\title{
Efficient S-Acylation of Thiourea
}

\author{
David J. Jones ${ }^{\mathrm{a}, \mathrm{b}, \mathrm{e}}$ \\ Uday B. Rao Khandavillia,b (D) \\ Eileen M. O'Leary \\ Simon E. Lawrence ${ }^{a, b, e}$ (D) \\ Timothy P. O'Sullivan*a,b,d (D) \\ a School of Chemistry, University College Cork, Cork, Ireland \\ tim.osullivan@ucc.ie \\ ${ }^{b}$ Analytical and Biological Chemistry Research Facility, Universi- \\ ty College Cork, Cork, Ireland \\ ' Department of Physical Sciences, Cork Institute of Technolo- \\ gy, Cork, Ireland \\ d School of Pharmacy, University College Cork, Cork, Ireland \\ e Synthesis and Solid-State Pharmaceutical Centre, University \\ College Cork, Cork, Ireland
}

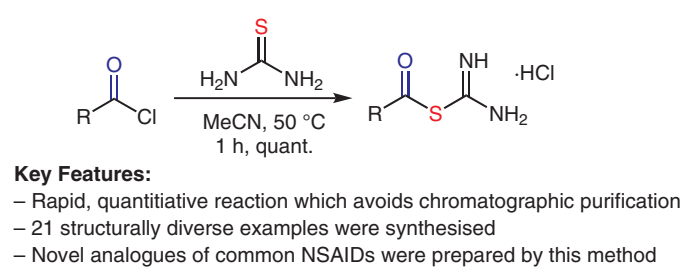

- 21 structurally diverse examples were synthesised

- Novel analogues of common NSAIDs were prepared by this method

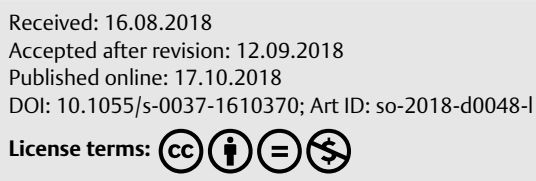

Abstract Efficient $S$-acylation of thiourea using a variety of acid chlorides is reported. Structurally diverse aryl and alkyl substrates are compatible with this methodology. Confirmation that acylation occurs exclusively on the sulfur atom of thiourea is provided by single-crystal $\mathrm{X}$-ray crystallographic analysis.

Key words isothiourea, thiourea, organosulfur, sulfur transfer

The thiourea motif is ubiquitous in organic chemistry. In particular, $S$-alkyl- and $S$-aryl isothioureas are widely employed as precursors to sulfur-containing heterocycles ${ }^{1}$ such as thiazoles ${ }^{2}$ and thiouracils. ${ }^{3}$ They are also useful in the preparation of sulfonyl chlorides, ${ }^{4}$ thiols, ${ }^{5}$ sulfides, ${ }^{6}$ disulfides $^{7}$ and guanidines. ${ }^{8}$ The sulfur transfer reagent 3mercaptopropionitrile is typically accessed from the corresponding isothiourea derivative. ${ }^{9}$ More recently, $\mathrm{S}$ - and $\mathrm{N}$ substituted thioureas have been incorporated into various organocatalysts to facilitate asymmetric induction through hydrogen bonding. ${ }^{10}$ Interestingly, isobenzofuran-substituted isothioureas undergo unusual base-mediated thermal rearrangement reactions to form isoindoles ${ }^{11}$ while isothiourea-substituted indolones rearrange under similar conditions to form thiazoles. ${ }^{12}$ The corresponding benzofuran-2-one analogues likewise rearrange to furnish 1,3-isothiazolidin-4-ones. ${ }^{13}$

$S$-Alkyl and $S$-aryl thioureas are notable molecules in their own right. They are known to inhibit a number of enzyme systems including nitric oxide synthase (NOS) $)^{14}$, monoamine oxidase (MAO) ${ }^{15}$ and $\mathrm{H} 3$ histamine receptors. ${ }^{16}$ Their inhibitory effect on multidrug resistant bacterial strains has also been described. ${ }^{17}$ Isothioureas have been found to disrupt biofilm formation in P. aeruginosa. ${ }^{18}$ Indol- amine 2,3-dioxygenase (IDO), an enzyme that is overexpressed in several disease states including cancer, is inhibited by a range of simple $S$-alkyl isothioureas. ${ }^{19}$ Allylic isothioureas have displayed potent antileukemia activity. ${ }^{20}$

While the formation of $S$-alkyl isothioureas is wellstudied, ${ }^{1 a, 1 c, 5 f, 20,21}$ there remains few systematic studies on the synthesis of $S$-acyl derivatives. It has previously been shown that thiourea reacts quantitatively with acetyl or benzoyl chloride to form the corresponding acylated thiourea adducts. ${ }^{22}$ However, it was not clear whether substitution had occurred on the sulfur or nitrogen atoms and these compounds were not characterised by modern techniques.

Herein, we report an efficient method for the selective $S$-acylation of thiourea, and we confirm that acylation occurs exclusively on the sulfur atom. Our method can be applied to a broad range of substrates, including the non-steroidal anti-inflammatory drugs (NSAIDs) Indomethacin, Aspirin, Diclofenac and Ibuprofen.

In the course of our studies on sulfur transfer reagents, we observed the formation of S-benzoyl isothiourea hydrochloride (2) on addition of benzoyl chloride (1) to a warm solution of thiourea in acetonitrile (Scheme 1 ).

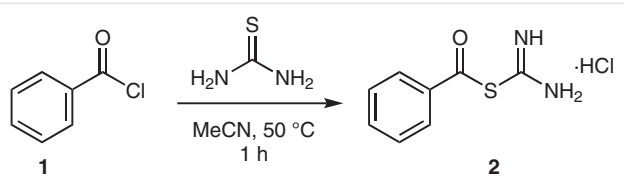

Scheme 1 Synthesis of S-benzoyl isothiourea hydrochloride (2)

The reaction proceeded rapidly with instantaneous precipitation of the product, which was isolated in quantitative yield without need for further purification. It was subsequently found that treatment of thiourea with a range of aryl-substituted acid chlorides furnished the corresponding 
S-acyl isothioureas 2-9 quantitatively (Scheme 2). The reaction tolerates aromatic acid chlorides with either electron-donating or electron-withdrawing substituents. This route also provides access to more sterically hindered ortho-substituted products, such as $\mathbf{4}$. Evidence that acylation had occurred exclusively on the sulfur atom, rather than on either of the nitrogen atoms, was provided by the apparent equivalence of the chemical shift values for the isothiouronium protons in the ${ }^{1} \mathrm{H}$ NMR spectrum of 2-9. No evidence for the formation of the $N$-acylated regioisomers was present in the spectra of the crude reaction mixtures. Additionally, recrystallisation of $\mathbf{5}$ and subsequent single-crystal Xray diffraction confirmed that acylation had indeed occurred at the sulfur atom (see the Supporting Information for details) (Figure 1). The equivalent $\mathrm{C}-\mathrm{N}$ bond lengths in $S$-acyl isothiourea $\mathbf{5}$ confirm the delocalization of the cation across the amidine portion of the molecule (Figure 2). These bond lengths are similar to those of previously described alkyl isothiourea $10 .{ }^{23}$ The S-C-N bond angle is smaller in $\mathbf{5}$ than in 10, perhaps reflecting the influence of the adjacent carbonyl group. The hydrogen bond network in $\mathbf{5}$ is constructed through bridging chloride ions via $\mathrm{N}-$ $\mathrm{H}$... Cl hydrogen bonding. Although the quaternary carbon signal of the isothiouronium group was not always apparent by ${ }^{13} \mathrm{C}$ NMR analysis, extending the relaxation delay from 1.00 second to 4.00 seconds allowed for the detection of the isothiouronium carbon in $\mathbf{2}$ (see the Supporting Information).

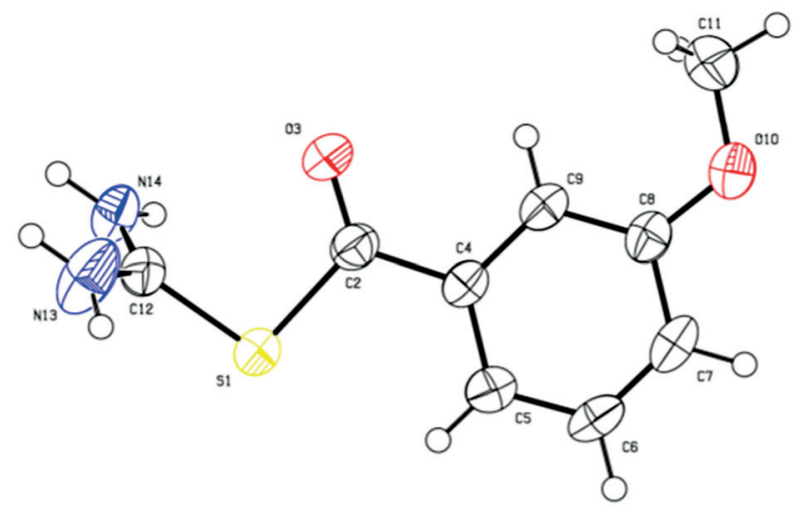

Figure 1 Crystal structure of 5, demonstrating that acylation has occurred at the sulfur atom

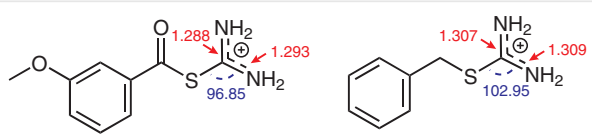

5

10

Figure 2 Comparison of bond lengths (in red) and S-C-N bond angles (in blue) between $\mathbf{5}$ and $\mathbf{1 0}^{23}$
Alkyl- and alkyl-aryl-substituted acid chlorides were subjected to the same conditions, affording 11-18 in quantitative yields (Scheme 3). As part of an ongoing effort in our laboratory to develop novel anti-inflammatory agents, we wondered whether this methodology could also be applied to some common NSAIDs. Diclofenac, Aspirin, Ibuprofen and Indomethacin were converted into their corresponding acid chlorides with oxalyl chloride in the presence of a catalytic amount of $\mathrm{N}, \mathrm{N}$-dimethylformamide. ${ }^{24}$ Addition of the resultant acid chlorides to a solution of thiourea in acetonitrile furnished NSAID derivatives 19-22, again in quantitative yields, with no apparent difference in reactivity (Scheme 4).
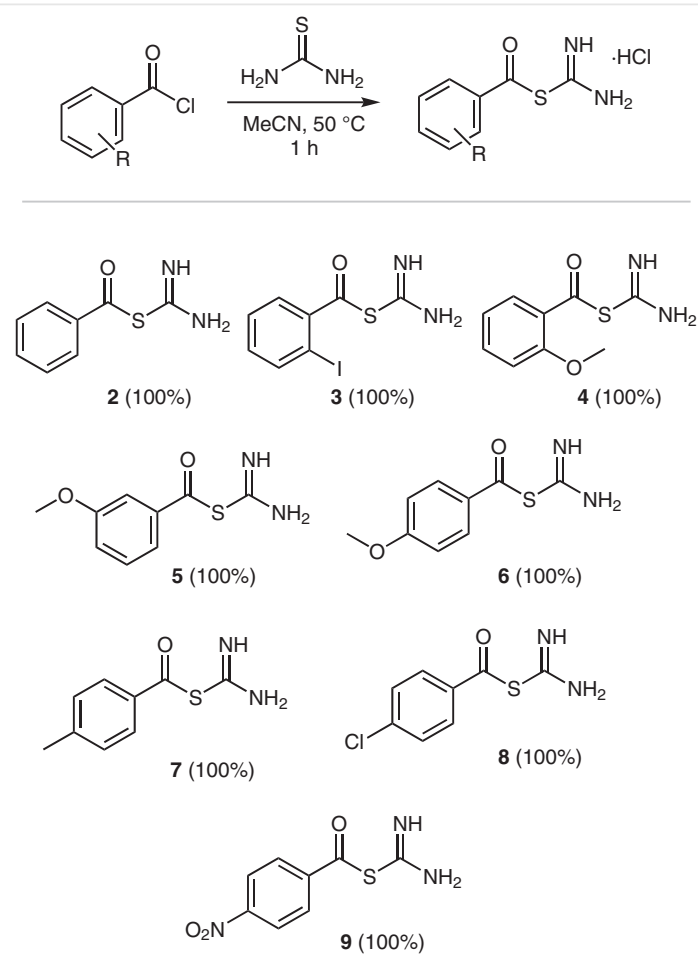

Scheme 2 Preparation of aryl-substituted S-acyl isothioureas 2-925

In conclusion, a method for the facile synthesis of $S$-acyl isothioureas has been described. The reactions proceed quantitatively, and the resulting solids are isolated without the need for further purification. We have shown that acylation occurs exclusively on the sulfur atom through crystallographic analysis. Several common NSAIDs were converted into their $S$-acyl isothiourea derivatives using this approach. 
.

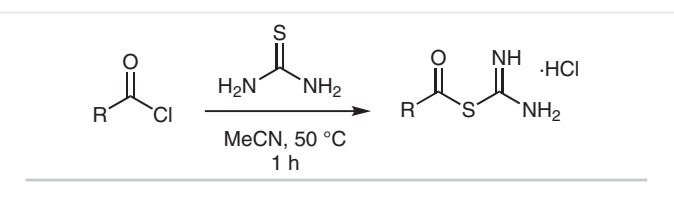

$\underbrace{N_{S}}_{11(100 \%)}$

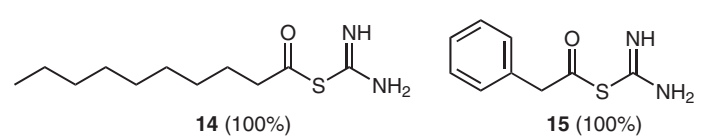<smiles>CCCCCCCCCCC(=O)SC(=N)N</smiles>

$16(100 \%)$

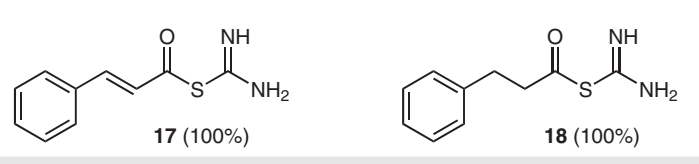

Scheme 3 Preparation of alkyl and aryl-alkyl derivatives 11-18 $\mathbf{2 5}^{25}$

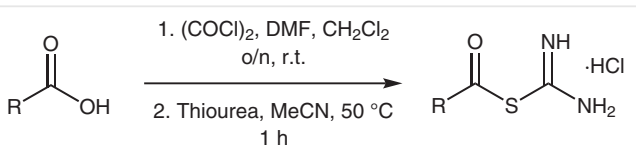
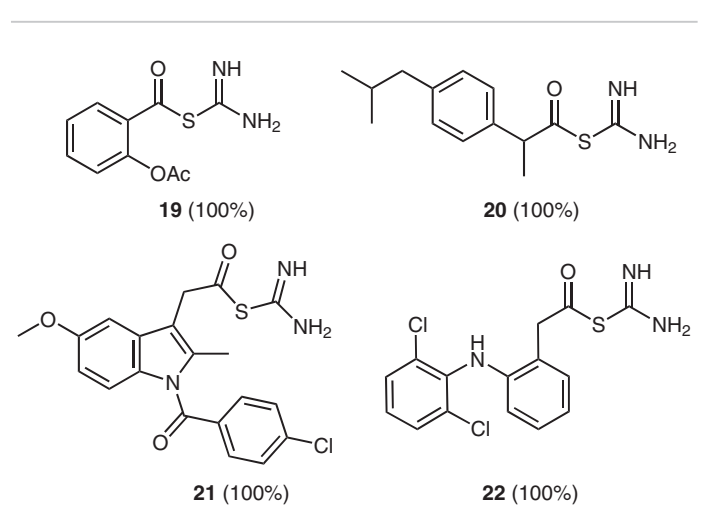

Scheme 4 Preparation of NSAID derivatives 19-225

\section{Funding Information}

This research received financial support from Science Foundation Ireland under Grant Numbers 12/RC/2275, 05/PICA/B802/EC07, 14/US/E2915 (UBRK) and the UCC Strategic Research Fund (UBRK and DJJ).

\section{Acknowledgment}

Dr Lorraine Bateman and Dr Denis Lynch are acknowledged for assistance with NMR spectroscopy.

\section{Supporting Information}

Details of the crystallographic analysis of compound $\mathbf{5}$ and full characterisation for all novel compounds with associated ${ }^{1} \mathrm{H}$ and ${ }^{13} \mathrm{C}$ NMR spectra can be found in the Supporting Information. The crystallographic data associated with this article have been deposited with the Cambridge Crystallographic Data Centre, CCDC 1586073. Supporting information for this article is available online at https://doi.org/10.1055/s-0037-1610370.

\section{References and Notes}

(1) (a) Krishnamurthy, M.; Basavaprabhu, ; Sharanabai, K. M.; Sureshbabu, V. V. Tetrahedron Lett. 2014, 55, 5609. (b) Kim, H.Y.; Kwak, S. H.; Lee, G.-H.; Gong, Y.-D. Tetrahedron 2014, 70, 8737. (c) Alcolea, V.; Garnica, P.; Palop, J.; Sanmartín, C.; González-Peñas, E.; Durán, A.; Lizarraga, E. Molecules 2017, 22, 1314. (d) Friebe, M.; Mahmood, A.; Spies, H.; Berger, R.; Johannsen, B.; Mohammed, A.; Eisenhut, M.; Bolzati, C.; Davison, A.; Jones, A. G. J. Med. Chem. 2000, 43, 2745. (e) Wu, Y.J.; Guernon, J.; Park, H.; Thompson, L. A. J. Org. Chem. 2016, 81, 3386.

(2) (a) Hickey, S. M.; White, J. M.; Pfeffer, F. M.; Ashton, T. D. Synlett 2015, 26, 1759. (b) Takaya, J.; Mio, K.; Shiraishi, T.; Kurokawa, T.; Otsuka, S.; Mori, Y.; Uesugi, M. J. Am. Chem. Soc. 2015, 137, 15859. (c) Xu, Z.; Guo, J.; Yang, Y.; Zhang, M.; Ba, M.; Li, Z.; Cao, Y.; He, R.; Yu, M.; Zhou, H.; Li, X.; Huang, X.; Guo, Y.; Guo, C. Eur. J. Med. Chem. 2016, 123, 309.

(3) (a) Palanki, M. S. S.; Erdman, P. E.; Manning, A. M.; Ow, A.; Ransone, L. J.; Spooner, C.; Suto, C.; Suto, M. Bioorg. Med. Chem. Lett. 2000, 10, 1645. (b) Zanatta, N.; Fortes, A. S.; Bencke, C. E.; Marangoni, M. A.; Camargo, A. F.; Fantinel, C. A.; Bonacorso, H. G.; Martins, M. A. P. Synthesis 2015, 47, 827. (c) Kaur, H.; Balzarini, J.; de Kock, C.; Smith, P. J.; Chibale, K.; Singh, K. Eur. J. Med. Chem. 2015, 101, 52. (d) Murai, K.; Miyazaki, S.; Fujioka, H. Tetrahedron Lett. 2012, 53, 3746.

(4) (a) Liu, C.; Guo, W.; Shi, X.; Kaium, M. A.; Gu, X.; Zhu, Y. Z. Eur. J. Med. Chem. 2011, 46, 3996. (b) Qiu, K.; Wang, R. Synthesis 2015, 47, 3186. (c) Yang, Z.; Zheng, Y.; Xu, J. Synlett 2013, 24, 2165.

(5) (a) Fujisaki, S.; Fujiwara, I.; Norisue, Y.; Kajigaeshi, S. Bull. Chem. Soc.Jpn. 1985, 58, 2429. (b) Murphy Kessabi, F.; Beaudegnies, R.; Quaranta, L.; Lamberth, C. Tetrahedron Lett. 2016, 57, 5511. (c) Floyd, N.; Vijayakrishnan, B.; Koeppe, J. R.; Davis, B. G. Angew. Chem. Int. Ed. 2009, 48, 7798. (d) Brown, T. J.; Chapman, R. F.; Cook, D. C.; Hart, T. W.; McLay, I. M.; Jordan, R.; Mason, J. S.; Palfreyman, M. N.; Walsh, R. J. A. J. Med. Chem. 1992, 35, 3613. (e) Minard, T. A.; Oswin, C. T.; Waldie, F. D. C.; Howell, J. K.; Scott, B. M. T.; Mondo, D. D.; Sullivan, R. J.; Stein, B.; Jennings, M.; Schlaf, M. J. Mol. Catal. A: Chem. 2016, 422, 175. (f) Zong, J.; Mague, J. T.; Pascal, Jr. R. A. Tetrahedron 2017, 73, 455. (g) Rotili, D.; De Luca, A.; Tarantino, D.; Pezzola, S.; Forgione, M.; Morozzo della Rocca, B.; Falconi, M.; Mai, A.; Caccuri, A. M. Eur. J. Med. Chem. 2015, 89, 156.

(6) (a) Su, X.; Pradaux-Caggiano, F.; Vicker, N.; Thomas, M. P.; Halem, H.; Culler, M. D.; Potter, B. V. L. ChemMedChem 2011, 6, 1616. (b) Marciniec, K.; Pawełczak, B.; Latocha, M.; Skrzypek, L.; Maciążek-Jurczyk, M.; Boryczka, S. Molecules 2017, 22, 300. (c) Lee, Y. S.; Kim, H. Y.; Kim, Y.; Seo, J. H.; Roh, E. J.; Han, H.; Shin, K. J. Bioorg. Med. Chem. 2012, 20, 4921. 
(7) (a) Emerson, D. W.; Bennett, B. L.; Steinberg, S. M. Synth. Commun. 2005, 35, 631. (b) Chukicheva, I. Y.; Sukrusheva, O. V.; Shumova, O. A.; Mazaletskaya, L. I.; Shevchenko, O. G.; Kuchin, A. V. Russ. J. Gen. Chem. 2016, 86, 2052. (c) Younai, A.; Fettinger, J. C.; Shaw, J. T. Tetrahedron 2012, 68, 4320.

(8) Aoyagi, N.; Furusho, Y.; Endo, T. Synlett 2014, 25, 983.

(9) (a) Jones, D. J.; O'Leary, E. M.; O'Sullivan, T. P. Tetrahedron Lett. 2017, 58, 4212. (b) Raz, R.; Rademann, J. Org. Lett. 2011, 13, 1606.

(10) (a) Najda-Mocarska, E.; Zakaszewska, A.; Janikowska, K.; Makowiec, S. Synth. Commun. 2018, 48, 14. (b) Supady, A.; Hecht, S.; Baldauf, C. Org. Lett. 2017, 19, 4199. (c) Serdyuk, O. V.; Heckel, C. M.; Tsogoeva, S. B. Org. Biomol. Chem. 2013, 11, 7051. (d) Fang, X.; Wang, C.-J. Chem. Commun. 2015, 1185. (e) Lippert, K. M.; Hof, K.; Gerbig, D.; Ley, D.; Hausmann, H.; Guenther, S.; Schreiner, P. R. Eur. J. Org. Chem. 2012, 5919.

(11) Váňa, J.; Sedlák, M.; Padělková, Z.; Hanusek, J. Tetrahedron 2012, 68, 9808.

(12) Kammel, R.; Tarabová, D.; Brož, B.; Hladíková, V.; Hanusek, J. Tetrahedron 2017, 73, 1861.

(13) Kammel, R.; Hanusek, J. Heterocycles 2014, 89, 1183.

(14) (a) Southan, G. J.; Szabo, C. Biochem. Pharmacol. 1996, 51, 383. (b) Southan, G. J.; Zingarelli, B.; O'Connor, M.; Salzman, A. L.; Szabo, C. Br. J. Pharmacol. 1996, 117, 619. (c) Southan, G. J.; Szabo, C.; Thiemermann, C. Br. J. Pharmacol. 1995, 114, 510. (d) Jang, D.; Szabo, C.; Murrell, G. A. Eur.J. Pharmacol. 1996, 312, 341.

(15) (a) Mostert, S.; Mentz, W.; Petzer, A.; Bergh, J. J.; Petzer, J. P. Bioorg. Med. Chem. 2012, 20, 7040. (b) Booysen, H. P.; Moraal, C.; Terre'Blanche, G.; Petzer, A.; Bergh, J. J.; Petzer, J. P. Bioorg. Med. Chem. 2011, 19, 7507.

(16) Ganellin, C. R.; Hosseini, S. K.; Khalaf, Y. S.; Tertiuk, W.; Arrang, J. M.; Garbarg, M.; Ligneau, X.; Schwartz, J. C. J. Med. Chem. 1995, $38,3342$.

(17) (a) Nicholson, A.; Perry, J. D.; James, A. L.; Stanforth, S. P.; Carnell, S.; Wilkinson, K.; Anjam Khan, C. M.; De Soyza, A.; Gould, F. K. Int. J. Antimicrob. Agents 2012, 39, 27. (b) Barker, C. A.; Allison, S. E.; Zlitni, S.; Nguyen, N. D.; Das, R.; Melacini, G.; Capretta, A. A.; Brown, E. D. Bioorg. Med. Chem. Lett. 2013, 23, 2426.

(18) (a) Bonez, P. C.; Rossi, G. G.; Bandeira, J. R.; Ramos, A. P.; Mizdal, C. R.; Agertt, V. A.; Dalla Nora, E. S. S.; de Souza, M. E.; dos Santos Alves, C. F.; dos Santos, F. S.; Gündel, A.; de Almeida Vaucher, R.; Santos, R. C. V.; de Campos, M. M. A. Microb. Pathog. 2017, 111, (Supplement C) 6. (b) Bonez, P. C.; Ramos, A. P.; Nascimento, K.; Copetti, P. M.; Souza, M. E.; Rossi, G. G.; Agertt, V. A.; Sagrillo, M. R.; Santos, R. C. V.; Campos, M. M. A. Microb. Pathog. 2016, 99, (Supplement C) 14.

(19) Matsuno, K.; Takai, K.; Isaka, Y.; Unno, Y.; Sato, M.; Takikawa, O.; Asai, A. Bioorg. Med. Chem. Lett. 2010, 20, 5126.

(20) Ferreira, M.; Assunção, L. S.; Silva, A. H.; Filippin-Monteiro, F. B.; Creczynski-Pasa, T. B.; Sá, M. M. Eur. J. Med. Chem. 2017, 129, (Supplement C) 151.

(21) (a) Jamaleddini, A.; Mohammadizadeh, M. R. Tetrahedron Lett. 2017, 58, 78-81. (b) Ingham, R. J.; Riva, E.; Nikbin, N.; Baxendale, I. R.; Ley, S. V. Org. Lett. 2012, 14, 3920. (c) Huang, Y.; Zhang, J.; Yu, Z.; Zhang, H.; Wang, Y.; Lingel, A.; Qi, W.; Gu, J.; Zhao, K.; Shultz, M. D.; Wang, L.; Fu, X.; Sun, Y.; Zhang, Q.; Jiang, X.; Zhang, J.; Zhang, C.; Li, L.; Zeng, J.; Feng, L.; Zhang, C.; Liu, Y.; Zhang, M.; Zhang, L.; Zhao, M.; Gao, Z.; Liu, X.; Fang, D.; Guo, H.; Mi, Y.; Gabriel, T.; Dillon, M. P.; Atadja, P.; Oyang, C. J. Med. Chem. 2017, 60, 2215. (d) Geies, A. A.; Abdel, Moneam. M. I.;
Kamal, El-Dean. A. M.; Zaki, R. M.; Abd, El-Naeem. E. E. J. Heterocycl. Chem. 2017, 54, 2012. (e) Mousdis, G. A.; Ganotopoulos, N.M.; Barkaoui, H.; Abid, Y.; Psycharis, V.; Savvidou, A.; Raptopoulou, C. P. Eur. J. Inorg. Chem. 2017, 3401. (f) Elacqua, E.; Manning, K. B.; Lye, D. S.; Pomarico, S. K.; Morgia, F.; Weck, M. J.Am. Chem. Soc. 2017, 139, 12240. (g) Abul-Futouh, H.; Almazahreh, L. R.; Harb, M. K.; Görls, H.; El-khateeb, M.; Weigand, W. Inorg. Chem. 2017, 56, 10437. (h) Manos-Turvey, A.; Al-Ashtal, H. A.; Needham, P. G.; Hartline, C. B.; Prichard, M. N.; Wipf, P.; Brodsky, J. L. Bioorg. Med. Chem. Lett. 2016, 26, 5087. (i) Tong, S.; Zhang, M.; Wang, S.; Yin, R.; Yu, R.; Wan, S.; Jiang, T.; Zhang, L. Eur. J. Med. Chem. 2016, 123, (Supplement C) 849.

(22) (a) Dixon, A. E.; Hawthorne, J.J. Chem. Soc., Trans. 1907, 91, 122. (b) Dixon, A. E.; Taylor, J. J. Chem. Soc., Trans. 1920, 117, 720.

(23) Hemalatha, P.; Veeravazhuthi, V. Acta Crystallogr., Sect. E: Struct. Rep. Online; 2008, 64 , 1805. CCDC Deposition Number: 695520.

(24) Biancalana, L.; Batchelor, L. K.; De Palo, A.; Zacchini, S.; Pampaloni, G.; Dyson, P. J.; Marchetti, F. Dalton Trans. 2017, 12001.

(25) General Procedure: To a stirred solution of thiourea (78 mg, 1.00 mmol, 1.00 equiv) in acetonitrile $(10 \mathrm{~mL})$ at $50{ }^{\circ} \mathrm{C}$ was added a solution of the required acid chloride ( $1.00 \mathrm{mmol}, 1.00$ equiv) in acetonitrile $(10 \mathrm{~mL})$ dropwise. The resulting thick suspension was allowed to stir at this temperature for a further one hour to ensure complete reaction. After one hour, the reaction mixture was cooled on ice and then vacuum filtered. The cake was washed with ethyl acetate $(2 \times 10 \mathrm{~mL})$ to afford the products. The products were obtained quantitatively unless otherwise stated.

\section{Representative Examples:}

$S$-(3-Methoxybenzoyl)isothiouronium Chloride (5)

Mp $166-168^{\circ} \mathrm{C}(\mathrm{MeCN})$.

${ }^{1} \mathrm{H}$ NMR $\left(400 \mathrm{MHz}\right.$, DMSO- $\left.d_{6}\right): \delta=3.80\left(\mathrm{~s}, 3 \mathrm{H}, \mathrm{OCH}_{3}\right), 7.19$ (dd, $J=8.15,1.83 \mathrm{~Hz}, 1 \mathrm{H}, \operatorname{ArC}(2) H), 7.39-7.44(\mathrm{~m}, 2 \mathrm{H}$, overlapping $\operatorname{ArC}(6) H$ and $\operatorname{ArC}(3) H), 7.53(\mathrm{~d}, J=8.15 \mathrm{~Hz}, 1 \mathrm{H}, \operatorname{ArC}(4) \underline{\mathrm{H}}), 9.62$ (bs, $\left.4 \mathrm{H}, \mathrm{NH}_{2}=\mathrm{C}-\mathrm{NH}_{2}\right) .{ }^{13} \mathrm{C}$ NMR $\left(100 \mathrm{MHz}\right.$, DMSO- $\left.d_{6}\right): \delta=55.2$, 113.8, 118.9, 121.5, 129.7, 132.1, 159.2, 167.1. IR (KBr): 3329, $3281,3163,3085,2954,2836,1697,1609,1583,1526,1470$, 1420, 1311, 1293, 1267, 1051, $755 \mathrm{~cm}^{-1}$. HRMS $\left(\mathrm{ESI}^{+}\right): \mathrm{m} / \mathrm{z}$ calcd. for $\mathrm{C}_{9} \mathrm{H}_{11} \mathrm{~N}_{2} \mathrm{O}_{2} \mathrm{~S}^{+}:$: 211.0536; found: 211.0528. Anal. Calcd. for $\mathrm{C}_{9} \mathrm{H}_{11} \mathrm{~N}_{2} \mathrm{O}_{2} \mathrm{ClS}$ : C, 44.00; H, 4.10; N, 11.40; Found: $\mathrm{C}, 44.31 ; \mathrm{H}$, $4.18 ; \mathrm{N}, 11.71$.

\section{$S$-(Decanoyl)isothiouronium Chloride (14)}

Mp $115-117^{\circ} \mathrm{C}(\mathrm{MeCN})$.

${ }^{1} \mathrm{H}$ NMR $\left(400 \mathrm{MHz}\right.$, DMSO- $\left.d_{6}\right): \delta=0.86(\mathrm{t}, J=7.51 \mathrm{~Hz}, 3 \mathrm{H}$, $\left.\mathrm{CH}_{3}(10)\right), 1.24\left(\mathrm{~m}, 12 \mathrm{H}, \mathrm{CH}_{2}(9-4)\right), 1.46-1.50\left(\mathrm{~m}, 2 \mathrm{H}, \mathrm{CH}_{2}(3)\right)$, $2.18\left(\mathrm{t}, J=7.88 \mathrm{~Hz}, 2 \mathrm{H}, \mathrm{CH}_{2}(2)\right), 9.18$ (bs, $\left.4 \mathrm{H}, \mathrm{NH}_{2}=\mathrm{C}-\mathrm{NH}_{2}\right) .{ }^{13} \mathrm{C}$ $\operatorname{NMR}\left(100 \mathrm{MHz}, \mathrm{DMSO}-d_{6}\right): \delta=13.9,22.1,24.5,28.5,28.6,28.7$, 31.2, 33.6, 174.5. IR (KBr): 3385, 3261, 3175, 3031, 2822, 2859, 1748, 1676, 1427, $732 \mathrm{~cm}^{-1}$. HRMS $\left(\mathrm{ESI}^{+}\right): \mathrm{m} / z$ calcd for $\mathrm{C}_{11} \mathrm{H}_{23} \mathrm{~N}_{2} \mathrm{OS}$ : 231.1531; Found: 231.1536. Anal. Calcd for $\mathrm{C}_{11} \mathrm{H}_{23} \mathrm{~N}_{2} \mathrm{OClS}$ : C, 49.52; H, 8.69; N, 10.50; Found: C, 49.18; H, 8.75; N, 10.71 .

\section{Indomethacin Analogue 21}

Mp $168-171{ }^{\circ} \mathrm{C}(\mathrm{MeCN})$.

${ }^{1} \mathrm{H}$ NMR (400 MHz, DMSO- $\left.d_{6}\right): \delta=2.22\left(\mathrm{~s}, 3 \mathrm{H}, \mathrm{ArCH}_{3}\right), 3.67(\mathrm{~s}$, $\left.2 \mathrm{H}, \mathrm{CH}_{2}\right), 3.76\left(\mathrm{~s}, 3 \mathrm{H}, \mathrm{OCH}_{3}\right), 6.72(\mathrm{dd}, J=8.18,1.33 \mathrm{~Hz}, 1 \mathrm{H}$, $\mathrm{C}(6) H), 6.92(\mathrm{~d}, J=8.18 \mathrm{~Hz}, 1 \mathrm{H}, \mathrm{C}(7) H), 7.05(\mathrm{~d}, J=1.33 \mathrm{~Hz}, 1 \mathrm{H}$, $\mathrm{C}(4) H)$. 7.64-7.70 (m, $4 \mathrm{H}, \operatorname{Ar}\left(2^{\prime}, 3^{\prime}, 5^{\prime}\right.$ and $\left.\left.6^{\prime}\right) H\right) .{ }^{13} \mathrm{C}$ NMR $(100$ MHz, DMSO- $\left.d_{6}\right): \delta=13.2,29.5,55.4,101.7,111.3,113.4,114.6$, 116.5, 129.0, 130.2, 130.71, 131.1, 134.1, 135.1, 137.6, 155.5, 
167.8, 172.0. IR (KBr): 3332, 3312, 2952, 1727, 1687, 1657, $1482,1308,1227,1047,792 \mathrm{~cm}^{-1}$. HRMS $\left(\mathrm{ESI}^{+}\right): \mathrm{m} / z$ calcd for $\mathrm{C}_{20} \mathrm{H}_{19} \mathrm{ClN}_{3} \mathrm{O}_{3} \mathrm{~S}^{+}$: 416.0830; Found: 416.0851. Anal. Calcd for $\mathrm{C}_{20} \mathrm{H}_{19} \mathrm{Cl}_{2} \mathrm{~N}_{3} \mathrm{O}_{3} \mathrm{~S}$ : C, 53.10; H, 4.23; N, 9.29; Found: C, 53.33; $\mathrm{H}$ 4.29; N, 9.45 